\title{
Three-Dimension Stress and Strain Distributions Across Five-Layer Human Aortic Wall
}

\author{
P. Khamdaengyodtai, P. Terdtoon, and P. Sakulchangsatjatai
}

\begin{abstract}
One of the implications of the structural changes in biomedical aspects is the change in stress and strain of the blood vessel. This research, therefore, aims to predict three-dimension stress and strain distributions across five-layer human aortic wall. In experiments, local aortic diameters can be obtained from cross-correlation technique on the ultrasound signal. Continuum mechanics is used as an approach to the results. The multilayer arterial wall is considered to be composed of five different layers. The three-dimensional effects are incorporated within the five-concentric axisymmetric layers while incorporating the nonlinear elastic characteristics under combined extension and inflation. Constitutive equation of fiber-reinforced material is employed for three major layers of intima, media and adventitia and constitutive equation of isotropic material is employed for other two layers of endothelium and internal elastic lamina. Relevant parameters for each layer are obtained by using nonlinear least square method fitted to in vivo experimental data on human aorta. Results from mechanical modeling, parameters could be precisely obtained with root of minimizes function of mean square error of pressures of $0.5631 \mathrm{kPa}$. Local stresses and strains distribution across deformed arterial wall could be illustrated and has been interpreted.
\end{abstract}

Index Terms-Fiber reinforced material, five layers artery, strain, stress, three dimensions.

\section{INTRODUCTION}

Circulatory system has the aortic vessel as an important part in carrying blood from pulsatile flow from the heart which acts as pump to steady flow in an organ. It is different from other engineering materials. Aortic vessels is biological tissue which not lies on Hooke's law thus the expression of the stress and strain on the non-linear elastic model has been demonstrated [1], [2]. Within the range of pressure considered in this study, the aortic vessel could be considered as incompressible material. According to this reason, extending the developed mechanical modeling from only one or two dimensions to completely three dimensions is possible. The heterogeneity of the aortic can be reasonable considered as multi homogeneous layers by histology of the aorta which simplifies complex constituents of the blood vessels to simple systemic [1], [3]. Moreover, the in vivo non-invasive imaging of ultrasound can be useful in the modeling. This research, therefore, aims to predict three-dimension stress

Manuscript received November 14, 2012; revised January 10, 2013.

Financial support is with the Thailand Research Fund through the Royal Golden Jubilee Ph.D.Program (PHD/0181/2549) to P. Khamdaengyodtai and P. Terdtoon is acknowledged.

P. Khamdaengyodtai, P. Terdtoon, and P. Sakulchangsatjatai are with Department of Mechanical Engineering, Faculty of Engineering, Chiang Mai University, 50200 Thailand (email: pannathai@hotmail.com, pradit@eng.cmu.ac.th,phrut235@yahoo.com). and strain distributions across five-layer human aortic wall.

\section{ANALYSIS}

\section{A. Experimental Data}

The pressure and diameter profile from experimental data for a human carotid artery [4] is shown in Fig. 1b and Fig. 1c. All experimental data are conducted by UEIL (Ultrasound and Elasticity Imaging Laboratory; UEIL, Biomedical Engineering and Radiology, Columbia University, NY, US). To obtain the diameter the ultrasound probe is placed on the skin at the carotid position [5]. The minima and maxima of the pressure and diameter waveforms are aligned and matched over the cardiac cycle. The viscosity effect is hence ignored.

\section{B. Continuum-Mechanical Framework}

The mathematical description of deformation [1], [2], the body occupy in the reference configuration $\Omega_{o}$. When the body is deformed, every particle at point $\boldsymbol{X}(R, \Theta, Z)$ transforms to new position at point $\boldsymbol{x}(r, \theta, z)$ in deformed configuration $\Omega$. The Green-Lagrange strain tensor can be determined from following equation. The transformation gradient could be determined as (1) where $\boldsymbol{C}$ denotes Right Cauchy Green tensor and $I$ denotes the second order unit tensor.

$$
\boldsymbol{E}=\frac{1}{2}(\boldsymbol{C}-\boldsymbol{I})
$$

For stress response of the artery, Cauchy stress tensor $\sigma$ relates to Green-Lagrange strain tensor $\boldsymbol{E}$ via transformation the Piola Kirchhoff stress tensor $S$ which is the first derivative $\partial \psi / \partial \boldsymbol{E}$ of strain energy function $\psi$ respected to Green-Lagrange strain tensor $\boldsymbol{E}$. Cauchy stress tensor $\boldsymbol{\sigma}$ could be expressed as (2) where $\boldsymbol{\sigma}_{v o l}=p \boldsymbol{I}$ and $p$ is Lagrange multiplier to descript incompressibility of artery wall.

$$
\sigma=\sigma_{v o l}+\bar{\sigma}
$$

\section{Mechanical Model}

Geometry and boundary conditions shown in Fig. 1a is in reference configuration. Kinematics of the artery in cylindrical coordinate, deformation equations [1] are as following.

$$
r=\sqrt{\frac{R^{2}-R_{i}^{2}}{k \lambda}+r_{i}^{2}}, \theta=k \Theta+Z \frac{\Phi}{L}, z=\lambda_{z} Z
$$




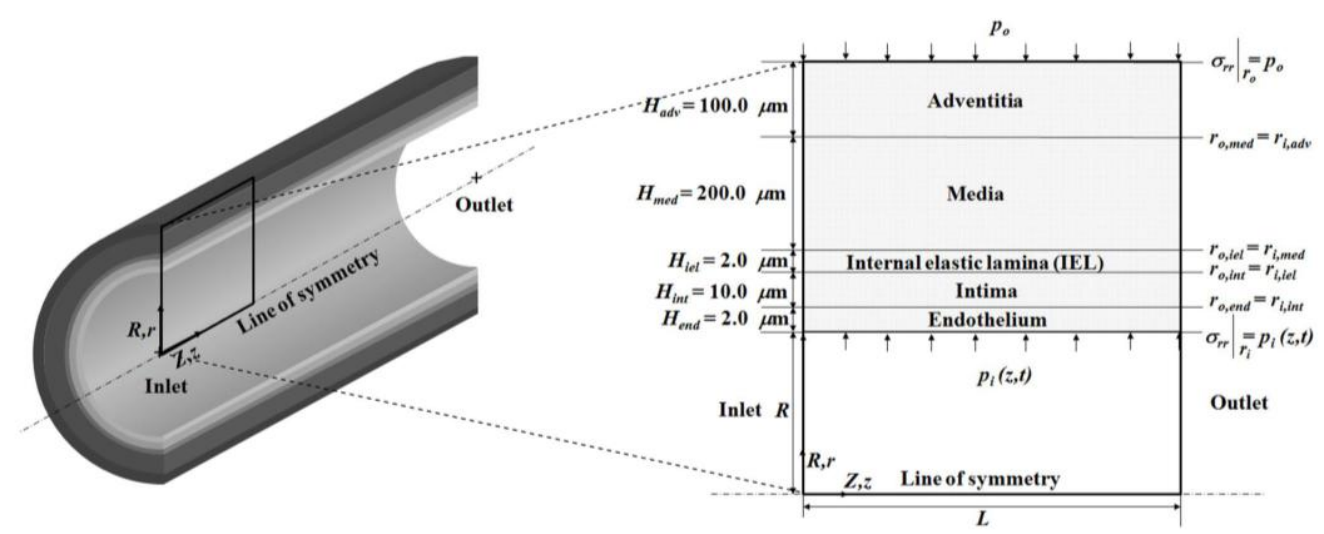

(a)

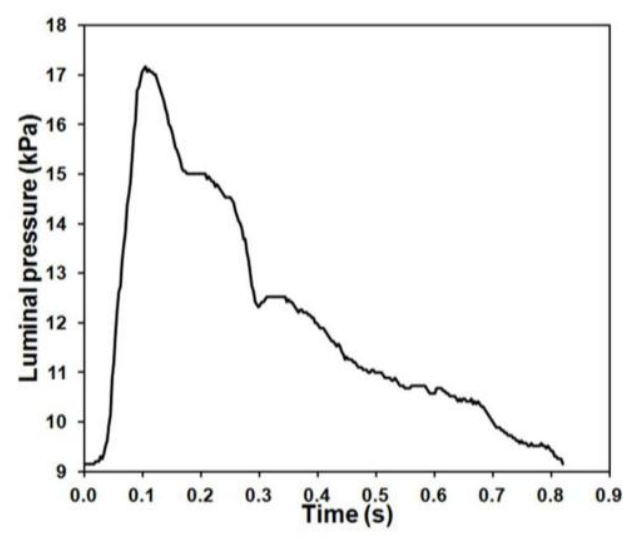

(b)

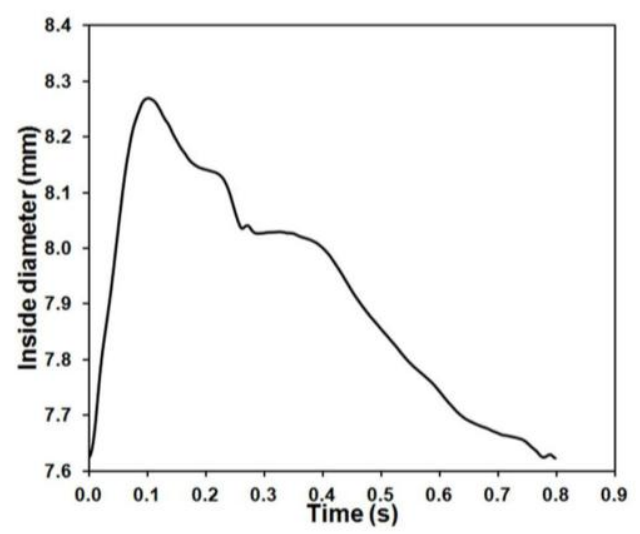

(c)

Fig. 1. (a) schematic illustration of an artery and the imposed boundary conditions, (b) the pressure profile along a cardiac cycle for a human carotid artery (male, time step $=0.140014 \mathrm{~ms}, N=5852$ points, cardiac cycle time $=$ time step $*(N-1)=0.8192 \mathrm{~s}$, heart rate $=73.240 \mathrm{bpm})$ and $(\mathrm{c})$ the diameter profile along a cardiac cycle for a human carotid artery (time step $=1 / 505 \mathrm{~ms}, N=404$ points, Cardiac cycle time $=$ Time step* $(N-1)=0.7980 \mathrm{sec}, \mathrm{Heart}$ rate $=75.186 \mathrm{bpm})$ $[5]$.

where $k=\frac{2 \pi}{2 \pi-\alpha}, \lambda_{z}$ is stretch ratio in longitudinal direction which is $1.1, \Phi, \alpha, L$ are twist angle, opening angle and overall length of artery in reference configuration respectively and subscript $i$ in (3) refers to inside. According to the artery structure, fiber reinforced strain energy function suggested by G.A. Holzapfel (2000) [1] had been suitable used. The strain energy function is expressed as (4) where $c_{j}>0, k_{1 j}>0$ are stress-like parameter and $k_{2 j}>0$ is dimensionless parameter, subscript $j$ refer to intima, media and adventitia layers and subscript $i$ refer to index number of invariants.

$$
\bar{\psi}_{j}=\frac{c_{j}}{2}\left(\bar{I}_{1}-3\right)+\frac{k_{1 j}}{2 k_{2 j}} \sum_{i=4,6}\left\{\exp \left[k_{2 j}\left(\bar{I}_{i j}-1\right)^{2}\right]-1\right\}
$$

For endothelium and internal elastic laminar (IEL), Equation 4 is considered only the first term. $\bar{I}_{1}$ and $\bar{I}_{i j}$ is invariant of $\boldsymbol{C}$. The stress in Eulerian description could be determined by the expression as

$$
\overline{\boldsymbol{\sigma}}_{j}=c_{j} \operatorname{dev} \overline{\boldsymbol{b}}+\sum_{i=4,6} 2 \bar{\psi}_{i j} \operatorname{dev}\left(\boldsymbol{a}_{i j} \otimes \boldsymbol{a}_{i j}\right)
$$

where $\bar{\psi}_{i j}, \boldsymbol{b}, \boldsymbol{a}_{i j}$ denotes response function, left Cauchy
Green tensor and Eulerian counter part of fiber direction vector. The equilibrium equation in cylindrical coordination is used with boundary conditions as shown in Fig. 1a. Moving boundary is normalized. Numerical integration with a three-point Gaussian quadrature is employed. Nonlinear least square method is used to estimate the relevant parameters by minimizing mean square error $M S E_{p a r}$ of luminal pressures given by (6).

$$
M S E_{p a r}=\frac{1}{N} \sum_{i=1}^{N}\left(p_{i, \text { mod el }}-p_{i, \text { exp eriment }}\right)^{2}
$$

\section{RESULTS AND DISCUSSION}

\section{A. Parameter Estimation}

Our computational model is compared to a number of prior studies [6] for one and two-layer material models by using their constitutive equations and material parameter sets in our in-house computational program. Parameters could be precisely obtained with root of minimizing function of mean square error of pressures of $0.5631 \mathrm{kPa}$. Using optimized parameter sets in Table I, the strain energy density contours in circumferential and longitudinal directions are investigated for each of the arterial layers. 
Diastolic blood pressure state $(69.61 \mathrm{mmHg})$
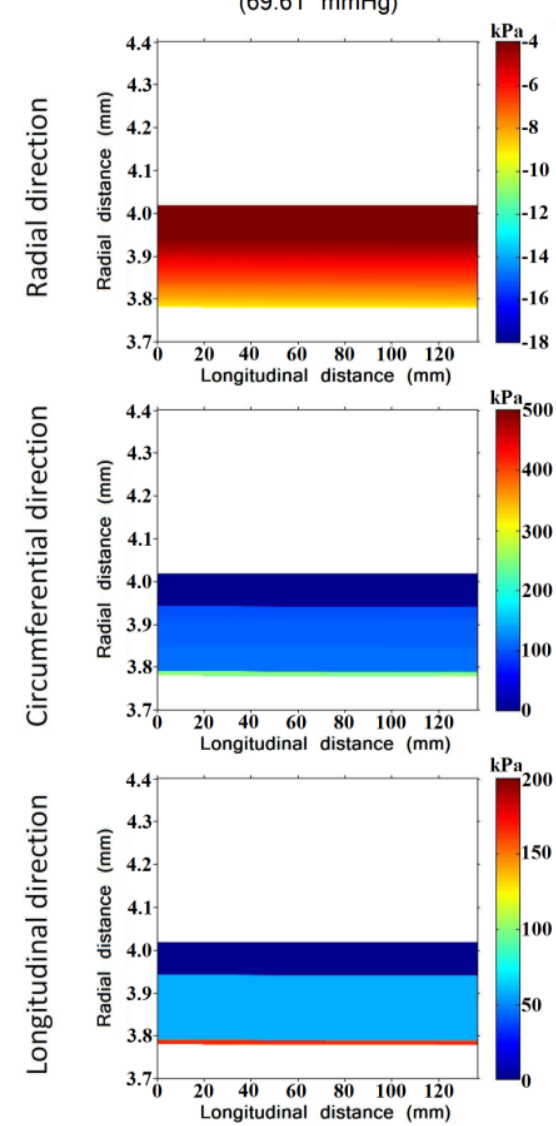

Mean blood pressure state $(89.25 \mathrm{mmHg})$
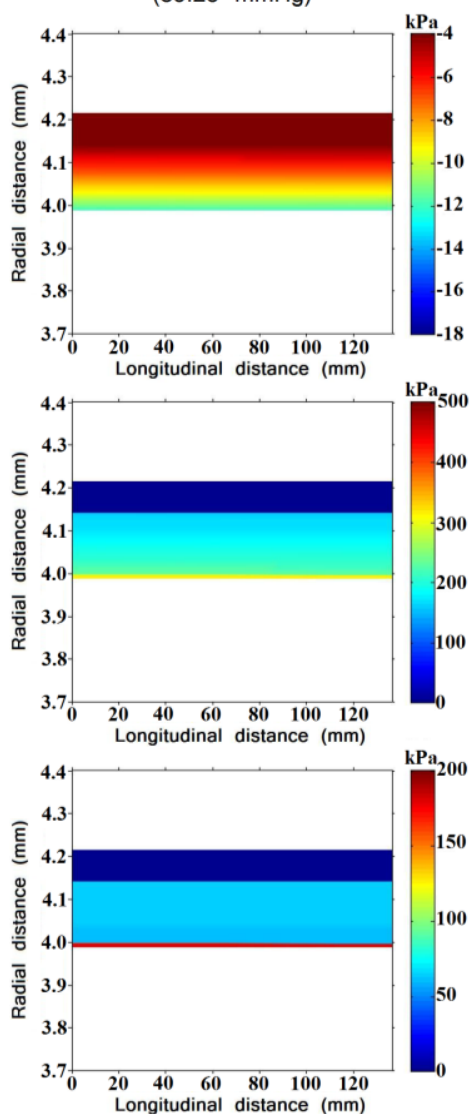

Systolic blood pressure state $(128.41 \mathrm{mmHg})$
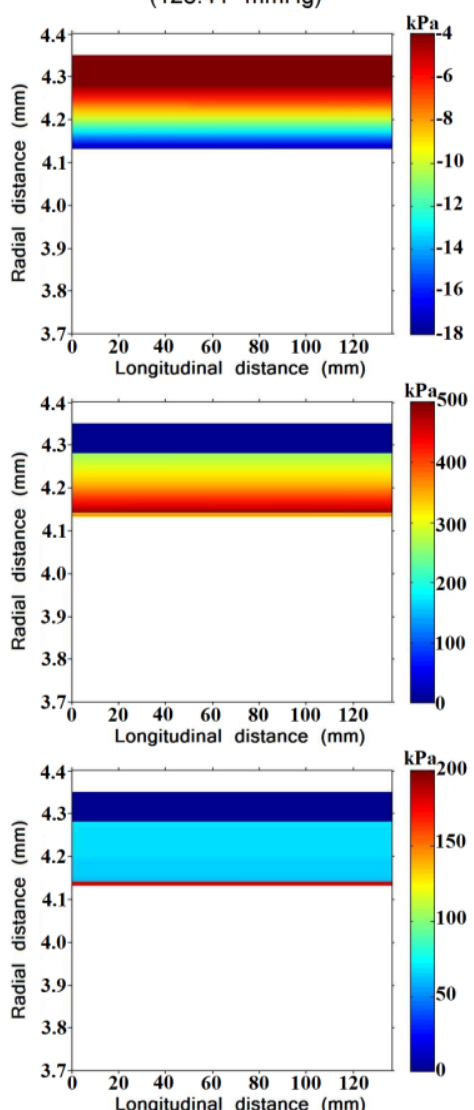

Fig. 2. Capture images of principal Cauchy stresses distributions across the $r-z$ plane deformed arterial wall.

Diastolic blood pressure state $(69.61 \mathrm{mmHg})$

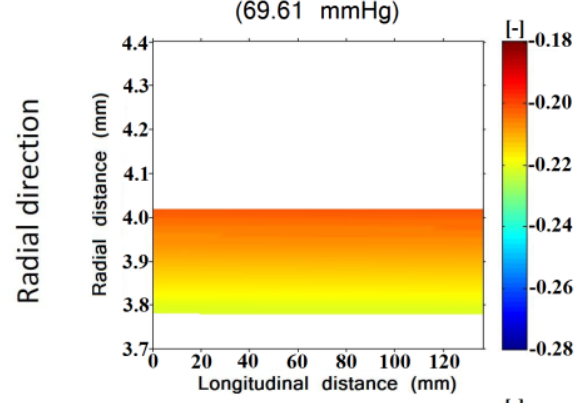

을
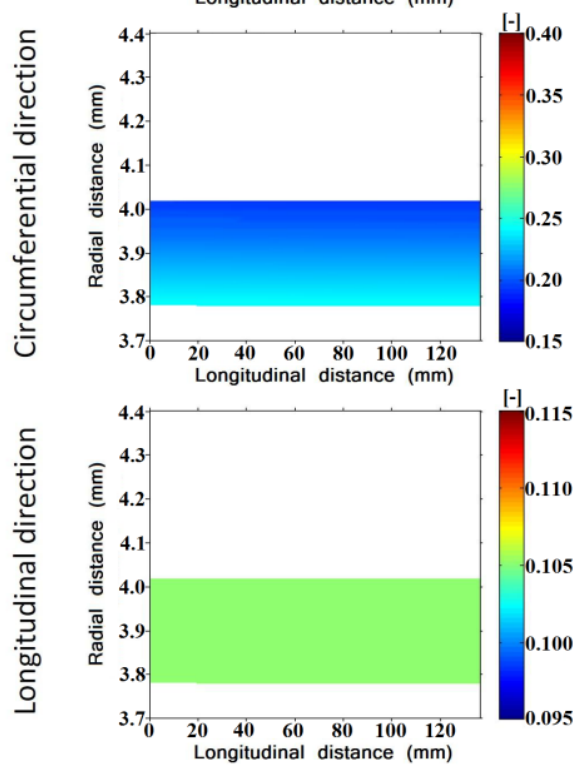

Mean blood pressure state $(89.25 \mathrm{mmHg})$
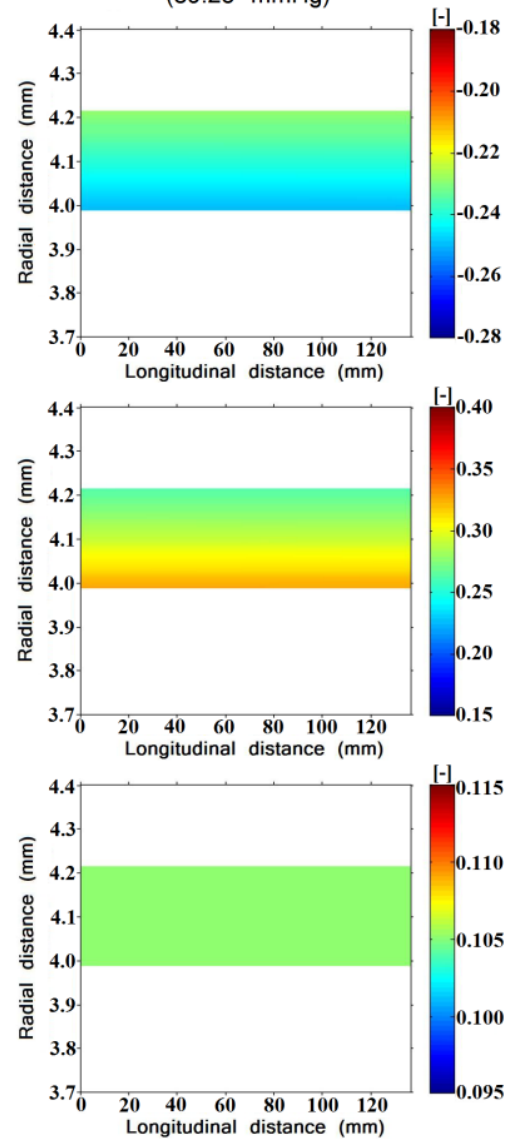

Systolic blood pressure state $(128.41 \mathrm{mmHg}$ )
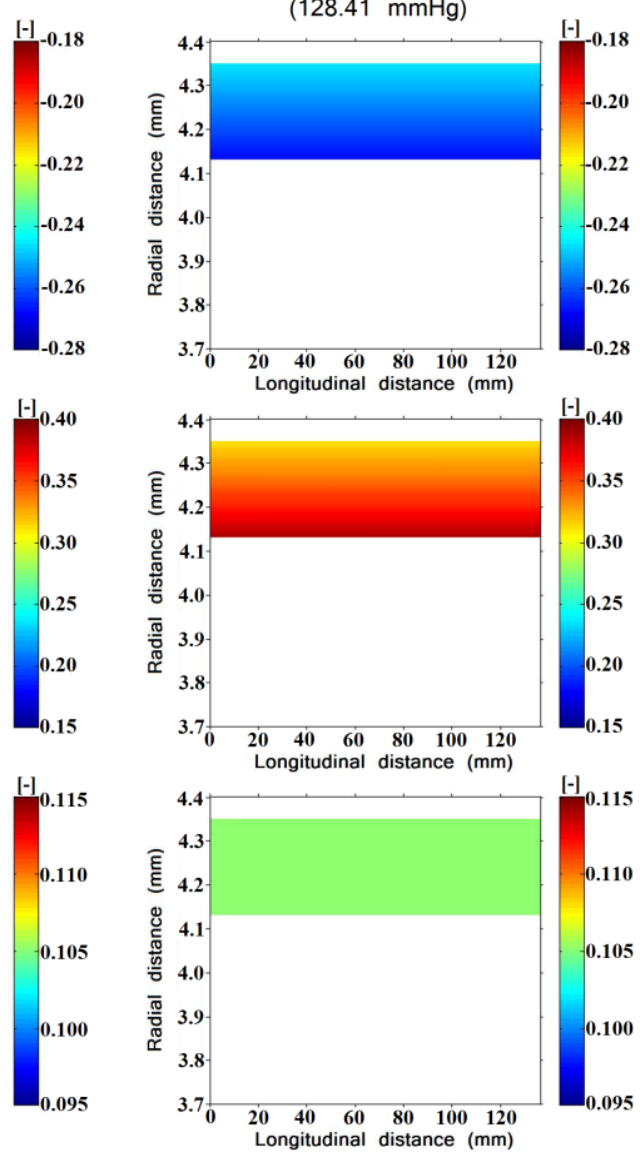

Fig. 3. Capture images of principal Green-Lagrange strains distributions across the $r-z$ plane deformed arterial wall. 


\section{B. Principal Cauchy Stresses and Green-Lagrange Strains Distributions across Aortic Wall}

Boundary conditions are investigated as luminal pressure and outside pressure are constrains of inside wall and outside wall. The consistent are obtained that could be seen in Fig. 2 in the radial stress distributions.

As illustrated in Fig. 2, magnitudes of principal Cauchy stresses of normal human artery in radial, circumferential and longitudinal directions are in range of 4.00-17.56 kPa, $3.96-493.80 \mathrm{kPa}$ and $2.18-184.56 \mathrm{kPa}$, respectively. The Cauchy stress in radial direction is continuous from inside wall which is equal to the negative value of luminal pressure and varies from this negative value at the inside wall and is continuous across the layers towards the pressure at the outside wall. The magnitude of the Cauchy stress in radial direction is smaller than in others directions (negative sign means it is compressive). In contrast to the Cauchy stress distributions in radial direction, the Cauchy stresses distributions in circumferential and longitudinal directions are discontinuous across the interfaces due to difference material properties of the layers which is also seen in previous studies [3], [7]. It can be seen that the circumferential stress in media layer is relatively high compared to the adventitia. The highest magnitude of circumferential stress occurs in intima. Overall the highest magnitude occurs around the inner wall and the lowest around the outer wall.

TABLE I: THE OPTIMIZED PARAMETERS

\begin{tabular}{lccc}
\hline \multirow{2}{*}{ Layer } & \multicolumn{3}{c}{ Optimized parameters } \\
\cline { 2 - 4 } & $c[\mathrm{kPa}]$ & $k_{1}[\mathrm{kPa}]$ & $k_{2}$ \\
\hline Endothelium & 250.9108 & - & - \\
Intima & 270.9837 & 2.1492 & 1.3012 \\
IEL & 250.9108 & - & - \\
Media & 100.3643 & 3.5820 & 5.2049 \\
Adventitia & 10.0364 & 0.0716 & 0.9759 \\
\hline \hline
\end{tabular}

As illustrated in Fig. 3, magnitude of principal Green-Lagrange strains of normal human artery in radial and circumferential directions are in range of $0.20-0.27$ and $0.19-0.39$, respectively and in longitudinal direction is equal to 0.1050 . The principal Green-Lagrange strain distributions in radial and circumferential directions possesses a higher magnitude at the inside walls and decreases continuously to a lower magnitude at the outside wall. The negative value of strain in radial direction signifies a reduction in the thickness of the arterial wall from the reference configuration. It can be seen that arterial wall becomes thinner as the luminal pressure increases. Stress and strain distributions are systematically synthesized in Fig. 2 and Fig. 3.

\section{CONCLUSION}

Three-dimension stress and strain distributions across five-layer human aortic wall can be predicted by using nonlinear constitutive equations suitably for each aortic layer. The endothelium and internal elastic lamina are treated as isotropic media and intima, media and adventitia are treated as anisotropic media incorporating the active collagen fibers. Layered arterial wall is modelled using two types of constitutive equations and based on in vivo experimental data.

\section{ACKNOWLEDGMENT}

The authors are also grateful to Professor Kambiz Vafai of Mechanical Engineering, University of California, Riverside CA, US, Associate Professor Elisa E. Konofagou of Ultrasound and Elasticity Imaging Laboratory, Biomedical Engineering and Radiology, Columbia University, NY, US and Graduate school of Chiang Mai University, TH.

\section{REFERENCES}

[1] G. A. Holzapfel, T. C. Gasser, and R. W. Ogden, "A new constitutive framework for arterial wall mechanics and a comparative study of material models," J. Elasticity, vol. 61, pp. 1-48, 2000.

[2] J. D. Humphrey, "Cardiovascular solid mechanics: cells, tissues, and organs," Springer-Verleg, pp. 757, 2002.

[3] P. Khamdaengyodtai, T. Khamdaeng, P. Sakulchangsatjatai, N. Kammuang-lue, and P. Terdtoon, "Stresses and strains distributions in three-dimension three-layer abdominal aortic wall based on in vivo ultrasound imaging," Journal of Science and Technology, Mahasarakham University, vol. 1, no. 5, 2012.

[4] T. Khamdaeng, J. Luo, J. Vappou, P. Terdtoon, and E. E. Konofagou, "Arterial stiffness identification of the human carotid artery using the stress-strain relationship in vivo," Ultrasonics, vol. 52, pp. 402-41, 2012 .

[5] J. Luo and E. E. Konofagou, "A fast normalized cross-correlation calculation method for motion estimation," IEEE Transactions on Ultrasonics, Ferroelectrics, and Frequency Control, vol. 57, no. 6, pp. 1347-1357, 2010

[6] P. Khamdaengyodtai, K. Vafai, P. Sakulchangsatjatai, and P. Terdtoon: "Effect of pressure on arterial failure," Journal of Biomechanics, vol. 45, pp. 2577-2588, 2012.

[7] G. A. Holzapfel, T. C. Gasser, and R.W. Ogden, "Comparison of a structural model with a Fung-type model using a carotid artery: Issues of material stability," The 1st GAMM Seminar on Continuum Biomechanics, vol. 2, no. 14, pp. 79-89, 2005.

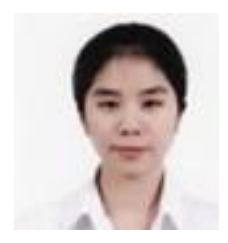

Pannathai Khamdaengyodtai is the $\mathrm{PhD}$ (Mechanical Engineering), Chiang Mai University, THAILAND and BEng (Mechanical Engineering) Chiang Mai University, Thailand.

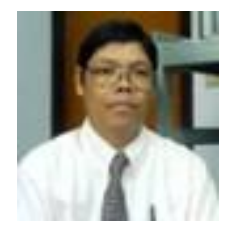

Pradit Terdtoon is the Prof. Dr./Dean of Graduate School, Mahasarakham University, THAILAND, Prof. Dr., Mechanical Engineering, Chiang Mai University, THAILAND, PhD (Mechanical Engineering), University of Tsukuba, JAPAN, MSc (Mechanical Engineering), Uni-versity of Reading, University, Thailand. UK, BEng (Mechanical Engineering), Chiang Mai

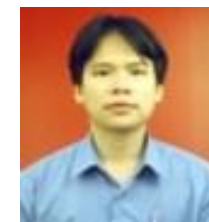

Phrut Sakulchangsatjatai is the Asst. Prof. Dr./Vice department Chairperson of Mechanical Engineering, Chiang Mai University, THAILAND, PhD (Mechanical Engineering) Chiang Mai University, THAILAND and BEng (Mechanical Engineering) Chiang Mai University, Thailand. 